\title{
Hydropower bidding strategies to day-ahead and real-time markets: different approaches
}

\author{
Yelena Vardanyan \\ KTH Royal Institute of Technology \\ Email: yelena.vardanyan@ee.kth.se
}

\author{
Lennart Söder \\ KTH Royal Institute of Technology \\ Email: lennart.soder@kth.se
}

\author{
Mikael Amelin \\ KTH Royal Institute of Technology \\ Email: amelin@ee.kth.se
}

\begin{abstract}
The ongoing growth in wind power introduce huge amount of uncertainties to the power market. The stochastic nature of these power sources increases the need for the reserve power in real-time market. Having a flexible power source, hydropower producer can provide reserve power and increase its profit. Therefore, to build a planning model, which will allocate available capacity in different market places is an essential task for the price-taker hydropower producer.

This paper uses optimal bidding model to the day-ahead market considering real-time balancing market under the uncertainties of the day-ahead and real-time market prices. Specifically, the model is built using stochastic linear programming approach. According to the results, for simultaneous bidding to day-ahead and real-time markets two extreme cases are happening. To make the bidding strategies more realistic and robust different novel approaches are modeled and assessed. Discussions on the results are provided and summarized.
\end{abstract}

Keywords-Stochastic programming, optimal bidding, dayahead market, regulating market, scenario generation

\section{INTRODUCTION}

Hydropower production has a big share of the total production mix in many electricity markets around the world [1]. Hydropower is considered a reliable and very flexible electric power source, which can change its production very fast and support to keep continuous balance between production and consumption in the electricity market [1].

There is a continuous increase in wind power production [2], which will increase production variation in the power system within short period. This production variation will increase the need for the reserve power and cause high volatile electric prices in real-time balancing market.

Being a flexible power source, hydropower can compensate wind power variation in the power system [3], [4]. Thus, hydropower producers need new advanced planning tools, which will allow to provide regulating power and earn extra profit. These tools have to be capable of working under competitive market conditions.

To have a more realistic production plan a hydropower producer has to consider uncertainties. A bidding strategy to day-ahead market for a price taker hydropower producer under uncertainty of the day-ahead market prices is developed in [5]. The author in [6] introduces multi-stage stochastic model under uncertainty of the spot market prices and water inflow. Bidding strategy to day-ahead market for price taker hydropower producer considering the possibility to trade on the intra-day market has been explained in [7]. In [8] and [9] the authors use stochastic dynamic programming to derive bidding curves A stochastic programming model for bidding to two-hour-ahead market is introduced in [10].

In addition, Olsson in [11] has introduced stochastic bidding model to day-ahead market considering real-time market. According to the results of this model, two extreme cases are tended to dominate in the decision making process; either the maximum amount is offered to up regulating market or the maximum amount is bidden to day-ahead market and put an offer on down regulating market. For a hydropower producer this is not a realistic case. Since day-ahead market sales are more definite, the hydropower producer will bid the base amount to day-ahead market and only small part will offer to real-time market.

This paper formulates different approaches, which force the optimization model to give more realistic solution. Each approach is a modification of the base optimization model, that creates bounds on the traded quantity on the real-time market. Every approach is assessed and discussed based on the results.

It is assumed that the hydropower producer is a price taker and doesn't have bilateral trading.

The paper is structured in the following way: section II represents planning model, section III describes method for time series analysis and develops forecasting model for the day-ahead and real-time market prices, section IV presents a case study and discusses different modeling approaches. Finally, section V concludes the paper.

\section{PlanNing MODEL}

The bids for all hours of the coming day to the day-ahead market are submitted at once. When the day-ahead market is cleared, day-ahead market prices for all hours of the next day are set at once. Then, so called second level uncertainty related to the development of the real-time market takes place. Rules for submitting bids to the real-time market are different. The real-time market for a specific hour is closed very short before the delivery. Hence, the real-time market prices are settled according to the time progress. As a producer of a 
flexible power source, a hydropower producer has to make optimal decision (to participate in the real-time market, or sell the whole electric power on the day-ahead market) under the uncertain data. Multistage stochastic programming technique is applicable, which is approximated by two-stage.

The first stage concerns the day-ahead bidding before the observation of the uncertain data, and the second stage contains bidding to real-time market and the actual dispatch. Probability description about the uncertain data is approximated by a so called scenario tree. The set $\left(\lambda_{s, t}, r_{s, t}^{\uparrow}, r_{s, t}^{\downarrow}\right)_{s \in S, t \in T}$, with corresponding probabilities $p_{s}$, generate this scenario tree.

The model is based on the hydropower planning model developed in [11] with some modifications. The start and stop cost of power plants, the forbidden discharge level and head dependency are not considered.

Let assume $s$ is an index for spot and real-time market price scenarios associated with $p_{s}$ probabilities. $t$ is time index and has hourly resolution and $T$ is the end of the planning period. $\lambda_{s, t}$ is price in spot market and $\lambda_{f}$ is water opportunity cost. $x_{s, t}$ is dispatch level to day-ahead market, $m_{j, T}^{s}$ is the end content of the reservoirs and $\gamma_{j}$ is expected future production equivalent for a plant. $r_{s, t}^{\uparrow}$ and $r_{s, t}^{\downarrow}, d_{s, t}^{\uparrow}$ and $d_{s, t}^{\downarrow}$ denote the prices and dispatch levels to up and down regulating market respectively. The objective function of the optimization problem applicable for power plants located in a row is presented below.

$$
\operatorname{Max} \sum_{s} p_{s}\left[\sum_{t}\left(\lambda_{s, t} x_{s, t}+r_{s, t}^{\uparrow} d_{s, t}^{\uparrow}-r_{s, t}^{\downarrow} d_{s, t}^{\downarrow}\right)+\lambda^{f} \sum_{j} \sum_{r \in R_{j}} \gamma_{r} m_{j, T}^{s}\right]
$$

The objective function (1) consists of expected profit from the day-ahead market, real-time market and future trading.

Both day-ahead market prices and dispatched volumes are unknown variables. To keep the model linear the possible bidding prices are fixed: equidistance price points $\rho_{i}$ are selected and the corresponding bid volumes $w_{i, t}$ are considered as variables. The Constraint presented below states the bidding rule to day-ahead market.

$$
x_{s, t}=\sum_{p=0}^{i} w_{i-p, t} \quad \text { if } \quad \rho_{i} \leq \lambda_{s, t} \leq \rho_{i+1},
$$

As it is necessary to keep the hydrological balance between reservoirs a constraint (3) is introduced: for each hour the reservoir's new contents $\left(m_{j, t}^{s}\right)$ are equal to its old contents $\left(m_{j, t-1}^{s}\right)$ plus water inflow $\left(I_{j, t}\right)$ minus water outflow. The discharge and the spillage $\left(S_{j-1, t-\tau_{j}}^{s}\right)$ from the upper reservoir flow in the reservoir located downstream with delay time $(\tau)$.

$$
\begin{array}{r}
m_{j, t}^{s}=m_{j, t-1}^{s}- \\
-\sum_{n} Q_{j, t, n}^{s}-S_{j, t}^{s}+\sum_{n} Q_{j-1, t-\tau_{j}, n}^{s} \\
+S_{j-1, t-\tau_{j}}^{s}+I_{j, t}, s \in S, t \in T,
\end{array}
$$

In addition, constraint (4) bounds hourly generation $G_{j, t}^{s}$ for each operated power plant according to its discharge volume $Q_{j, t, n}^{s}$ and marginal production equivalent.

$$
G_{j, t}^{s} \leq \sum_{n} \mu_{j, n} Q_{j, t, n}^{s}
$$

The quantity traded on the day-ahead and real-time markets should be equal to the total output of the generation units for each hour. This leads to the constraint (5).

$$
x_{s, t}+d_{s, t}^{\uparrow}-d_{s, t}^{\downarrow}=\sum_{j} G_{j, t}^{s} ;
$$

The amount of energy traded on downward balancing market can not be larger than the amount of energy traded on the day-ahead market. This is provided by (6).

$$
d_{s, t}^{\downarrow} \leq x_{s, t} ;
$$

Last two constraints together put bounds on hourly discharge level and on hourly water storage level respectively.

$$
\begin{gathered}
Q_{j, t, n}^{s} \leq \bar{q}_{j, n}, \\
m_{j, t}^{s} \leq \bar{m}_{j},
\end{gathered}
$$

Moreover, all variables $\in R_{+}, \bar{q}_{j, n}$ and $\bar{m}_{j}$ are maximum discharge level and maximum reservoir content respectively.

\section{TIME SERIES ANALYSIS}

One of the commonly used forecasting techniques to analyze day-ahead market price time series is Autoregressive Integrated Moving Average (ARIMA) [12]. ARIMA model is used to forecast day-ahead market prices and to generate scenarios based on that. Then SARIMA time series analysis tool together with Markov chain is used to forecast real-time market prices and to generate corresponding price scenarios [13]. Time series used for day-ahead and real-time price forecast are historical data for 2011 published on Nord pool.

\section{A. Day-ahead market price model}

Non-stationary series is provided by the ARIMA processes, which reduces to ARMA process after differencing finitely many times [14], [15]. The $\operatorname{ARMA}(p, q)$ process can be expressed in the following form: $\phi(B) \lambda_{t}=\theta(B) \varepsilon_{t}$; where $\lambda_{t}$ is the day-ahead market price at time t, $\phi$ and $\theta$ are respectively p-th and q-th degree polynomials

$$
\left.\begin{array}{c}
\phi(B)=1-\phi_{1} B-\ldots-\phi_{p} B^{p}, \\
\theta(B)=1+\theta_{1} B+\ldots+\theta_{q} B^{q},
\end{array}\right\}
$$

and $\mathrm{B}$ is backward shift operator defined by $B^{j} \lambda_{t}=\lambda_{t-j}$. In addition, $\varepsilon_{t}$ is white noise sequence, which is independently and identically distributed with 0 mean and $\sigma^{2}$ variance: $\varepsilon_{t} \sim$ $W N\left(0, \sigma^{2}\right)$.

The following steps have to be completed to construct a model which best fits the time series: identification, estimation, validation and prediction. Identification process is curried out studying the ACF and PACF of the historical time series.

A preliminary model is build and the corresponding parameters are estimated according to Maximum Likelihood (ML) 
method. Then the model is refined studying the autocorrelation (ACF) and partial autocorrelation (PACF) functions of the residuals; weather they resemble a white noise. In addition, the Ljung-Box statistics is applied, according to which, if $H=0$ the hypothesis that the residuals resemble white noise series can't be rejected. If according to ACF, PACF and Ljung-Box statistics the residuals series do not fulfill the requirements to be a white noise, the model orders $(p, q)$ are adjusted and the whole procedure is repeated again.

The constructed ARMA model ready for day-ahead market price prediction is the following.

$$
\begin{array}{r}
\left(1-\phi_{1} B^{1}\right)\left(1-\phi_{17} B^{17}-\phi_{23} B^{23}-\phi_{48} B^{48}-\phi_{72} B^{72}\right. \\
\left.-\phi_{167} B^{167}\right) \log \lambda_{t}=\left(1-\theta_{1} B^{1}-\theta_{2} B^{2}-\theta_{3} B^{3}\right. \\
\left.-\theta_{4} B^{4}-\theta_{5} B^{5}\right)\left(1-\theta_{18} B^{18}-\theta_{45} B^{45}-\theta_{68} B^{68}\right) \varepsilon_{t}
\end{array}
$$

\section{B. Regulating market price model}

The day-ahead market prices serve a bound for real-time market prices: upward regulated prices are always greater than day-ahead market prices and downward regulated prices are always less than day-ahead market prices. It is unusual to have both upward and downward regulation happening within one hour. Most of the cases either upward or downward regulation is taking place.

As it is mentioned already the combination of SARIMA and Markov processes is used to model real-time market prices. In detail description of the topic see [13].

1) SARIMA model: (11).

The general form of the SARIMA process is expressed in

$$
\Phi\left(B^{\beta}\right) \phi(B) \Delta_{\beta}^{D} \Delta^{d} \ln \left(\delta_{t}\right)=\Theta\left(B^{\beta}\right) \theta(B) \varepsilon_{t}
$$

where $\Delta_{\beta}^{D}=\left(1-B^{\beta}\right)^{D}, \Delta^{d}=(1-B)^{d}$ and $\Phi\left(B^{\beta}\right), \phi(B), \Theta\left(B^{\beta}\right)$ and $\theta(B)$ are polynomials expressed as in (12), (13) and $\varepsilon_{t} \in$ $N\left(0, \sigma^{2}\right)$ is white noise sequence.

$$
\left.\begin{array}{c}
\Phi\left(B^{\beta}\right)=1-\Phi_{1} B^{\beta}-\Phi_{2} B^{2 \beta}-\ldots-\Phi_{P} B^{P \beta}, \\
\Theta\left(B^{\beta}\right)=1+\Theta_{1} B^{\beta}+\Theta_{2} B^{2 \beta}+\ldots+\Theta_{Q} B^{Q \beta},
\end{array}\right\}
$$

Lets denote $\delta_{t}$ as a difference between day-ahead market prices and real-time market prices for each hour (14). In order to reflect strong correlation between the spot market prices and the real-time market prices their difference, $\delta_{t}$ is logarithmized and modeled

$$
\delta_{t}=a_{t}-\lambda_{t}
$$

Then, model orders are set and parameters are estimated. SARIMA models for upward and downward regulation prices are presented below:

- The upward regulation prices are modeled with a SARIMA $(1,0,1) \times(1,0,1)_{24}$ process expressed by the following polynomials:

$$
\left.\left.\begin{array}{l}
\Phi(B)=1-0.023 B^{24}, \\
\Theta(B)=1+0.009 B^{24},
\end{array}\right\} \begin{array}{l}
\phi(B)=1-0.63 B, \\
\theta(B)=1+0.09 B,
\end{array}\right\}
$$

- The downward regulation prices are modeled with the SARIMA $(1,0,1) \times(1,0,1)_{23}$ process expressed by the following polynomials:

$$
\left.\left.\begin{array}{l}
\Phi(B)=1-0.013 B^{23}, \\
\Theta(B)=1-0.006 B^{23},
\end{array}\right\} \begin{array}{l}
\phi(B)=1-0.43 B, \\
\theta(B)=1+0.15 B,
\end{array}\right\}
$$

The models are validated using the same methods as for the ARIMA model case.

2) Markov model:

To have a Markov model to describe the discrete behavior of the system for next 24 hours, first the probabilities of the transition matrix has to be estimated. Those parameters can be estimated using historical market prices. Based on the price series, $\left(b_{t}^{\uparrow}, b_{t}^{\downarrow}\right)$ pairs are obtained, which show the state of the upward and downward prices at time $t$. Let $o_{t}$ be the variable which show the price state at time $t$ as it is demonstrated below

$$
o_{t}=\left\{\begin{array}{lll}
1, & \text { if } & \left(b_{t}^{\uparrow}, b_{t}^{\downarrow}\right)=(0,0) \\
2, & \text { if } & \left(b_{t}^{\uparrow}, b_{t}^{\downarrow}\right)=(0,1) \\
3, & \text { if } \quad\left(b_{t}^{\uparrow}, b_{t}^{\downarrow}\right)=(1,0) \\
4, & \text { if } \quad\left(b_{t}^{\uparrow}, b_{t}^{\downarrow}\right)=(1,1)
\end{array} \quad t=1,2 \ldots T\right.
$$

Let $O_{i j}^{h}$ be set defined in the following way

$$
O_{i j}^{h}=o_{t}: o_{t}=j, o_{t-1}=i, \ldots, o_{t-h}=i, t=h+1, \ldots, T
$$

then transition probabilities for $i, j=1, \ldots, 4$ can be calculated like in (19)

$$
p_{i j}^{h}=\frac{\# O_{i j}^{h}}{\sum_{n=1}^{4} \# O_{i, n}^{h}}, \quad i, j=1, \ldots 4
$$

The obtained transmission matrix is shown in (20)

$$
P=\left(\begin{array}{cccc}
0.6396 & 0.1762 & 0.1816 & 0.0054 \\
0.1052 & 0.8259 & 0.0414 & 0.0276 \\
0.1452 & 0.0456 & 0.7805 & 0.0269 \\
0.0645 & 0.4516 & 0.4839 & 0
\end{array}\right)
$$

Having transmission probability matrix estimated, it is possible to predict different scenarios for the state of the system for coming 24 hours.

\section{CASE STUdy}

For the case study three cascaded reservoirs are studied. Each Each reservoir has one power station.

Table I summarizes the maximum storage capacity, the maximum design flow, the maximum production capacity for each power plants and water delay time.

The models described in section III are used to predict market prices. The optimization model presented in section II is programmed in GAMS and CPLEX solver is used to solve it. All assumptions are listed below:

- The future electricity price is considered deterministic setting 50 Euro/MWh and is estimated taking the average 
Table I

DATA FOR POWER PLANTS

\begin{tabular}{|l|l|l|l|l|}
\hline & $\bar{m}_{j}(\mathrm{HE})$ & $\bar{Q}_{j}\left(m^{3} / s\right)$ & $\bar{G}_{j}(\mathrm{MW})$ & $\tau_{j}(\mathrm{~h})$ \\
\hline I & 305856 & 340 & 95 & 0 \\
\hline II & 1392 & 310 & 50 & 0.5 \\
\hline III & 4008 & 330 & 90 & 2 \\
\hline
\end{tabular}

value of futures and forward contracts.

- Initial reservoir content is considered $36 \%$ full.

- Ten day-ahead market price scenarios are generated for each hour based on the day-ahead market price forecast.

- For each day-ahead market price scenario three upward regulated and three downward regulating price scenarios are generated.

The simulated result gives bidding volumes to different market places for each hour Figure 1.

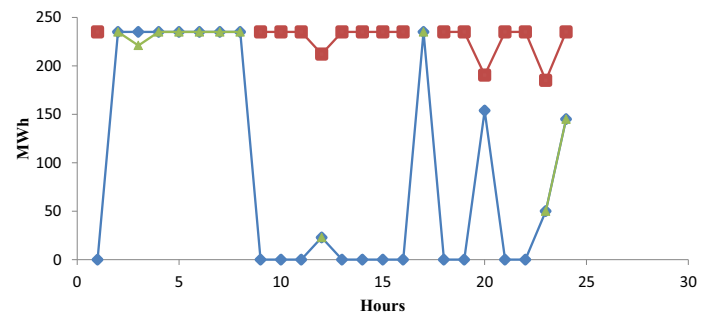

Figure 1. Base case: $\diamond$ represents traded spot volume, $\square / \triangle$ traded up/down regulation volume.

According to Figure 1 for most of the hours the following happens:

- The maximum amount is bidden to upward regulating market

- The maximum amount is bidden to day ahead market and offered to buy back in downward regulating market

Similar results have been observed for example in [11]. The solution is optimal; however the solution is not realistic. It is expected that, the hydropower producer for each hour will bid the main volume to day-ahead market and only small quantity will offer to up or down regulating market. This behavior is dependent on the special characteristics of dayahead market and real-time market. Sales on day-ahead market are definite; when a bid is accepted on day-ahead market, the quantity mentioned on the bid must be provided. In contrast, sales on real-time market are uncertain. Bids submitted to the regulating power market in this paper concern as an energy volume in MWh. These bids are activated by the System Operator, when the system needs regulating power. Thus, the amount of regulating power the actor will buy or sell totally depends on the duration the bid has been active within that hour. The remaining volume has to be stored for the future usage. If this happen in several consecutive hours, it can cause spillage for a system with a small storing capacity.

The different modifications of the base model are following.
Trivial approach The first approach is very trivial. It states that the maximum bidden amount to the up/down regulation cannot be higher than $20 \%$ of the maximum capacity. The result is as naive as the approach itself; whenever it is beneficial to participate in upward/downward regulation market, it bids the allowed amount $(20 \%)$ and remaining amount bids to day ahead market Figure 2.

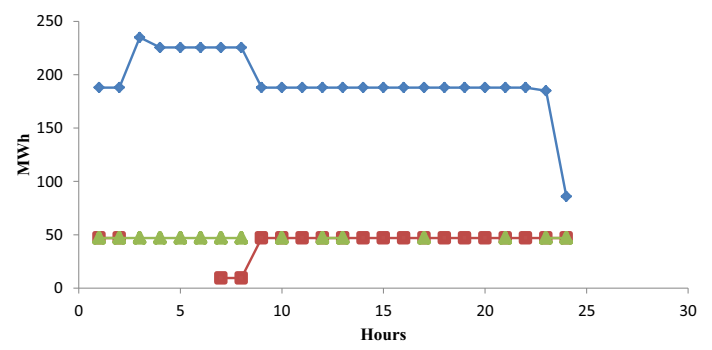

Figure 2. Trivial case: $\diamond$ represents traded spot volume, $\square / \triangle$ traded up/down regulation volume.

Limit the end reservoir content approach The second approach is fixing the reservoirs content at the end of the planning period. The results give more flexible solution, but still it is not very realistic Figure 3.

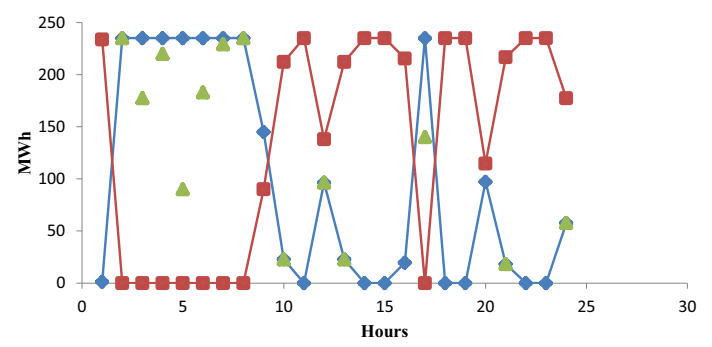

Figure 3. Fixing the end content of reservoirs case: $\diamond$ represents traded spot volume, $\square / \triangle$ traded up/down regulation volume.

According to Figure 3, up/down regulation volume is less than the maximum capacity for most of the hours. Generally speaking, overall results get better, but the improvement is not sharp.

Non-linear approach In the third approach we have assumed that real-time market prices are highly influenced by the bid volume. The real-time market prices are least beneficial (low for upward regulation and high for downward regulation), when the bid volume is equal to the maximum capacity and improving gradually while bid volume is decreasing. With the introduction of variable real-time market prices the objective function becomes nonlinear and nonconvex. Thus, it is not possible to claim that the local optimum is also a global optimum.

Figure 4 shows the results. As one can see the results are more realistic in this case: for every hour the base volume is traded on day-ahead market and only small amount is bid to upward or downward regulated market. 


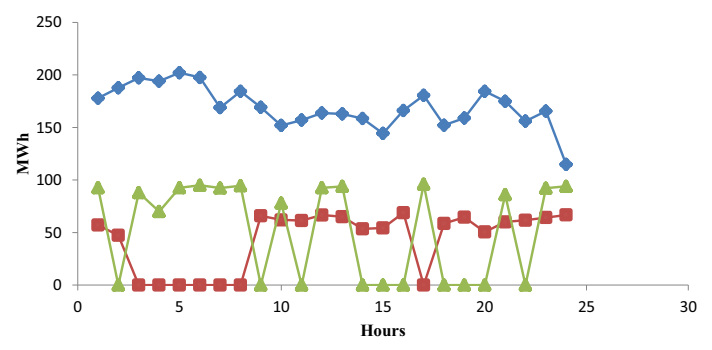

Figure 4. Nonlinear case: $\diamond$ represents traded spot volume, $\square / \triangle$ traded up/down regulation volume.

The main drawbacks of this approach are that it is not easy to prove the global optimality of the solution and it takes 10 hours to solve.

Discretized approach The last approach is the advanced version of the trivial approach. In this case we assume that available traded amount to upward/downward market has upper bound (20\% of the maximum capacity). However, upward/downward market prices are discretized and different percentage of the available amount is bid according to that. For example, if upward/downward market prices are not very attractive it bids only $10 \%$ of the available quantity. It increases the bidding percentage when prices get better and finally it bids all volume only for the case when the prices are the most beneficial. The results are drawn in the Figure 5.

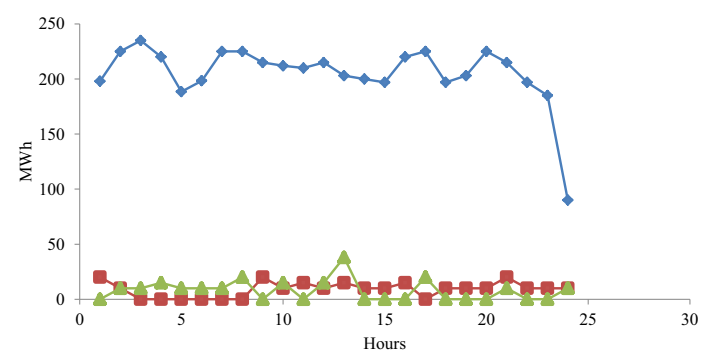

Figure 5. Discretized case: $\diamond$ represents traded spot volume, $\square / \triangle$ traded up/down regulation volume.

In this case also the base quantity is traded in the day-ahead market. Small amount is traded on the upward/downward market. The model is linear and solving time is very short. Table II summarizes different approaches.

From all cases above the most promising and applicable approach is the fourth one: it gives flexible results, keeps the optimization problem linear and it is fast to solve.

\section{CONCLUSION}

The model used in this paper is an optimal bidding strategy to the day-ahead market while considering real-time market. The results of this model are not realistic. They tend to follow two dominant trends: either to participate only in upward regulating market or sell the whole capacity in dayahead market and put an offer to bring back in downward
Table II

SUMMARY OF THE RESULTS

\begin{tabular}{|l|l|l|l|}
\hline Approaches & $\begin{array}{l}\text { Model } \\
\text { type }\end{array}$ & Results & $\begin{array}{l}\text { Solution } \\
\text { time }\end{array}$ \\
\hline Base case & Linear & $\begin{array}{l}\text { Optimal but not re- } \\
\text { alistic }\end{array}$ & $\begin{array}{l}12 \\
\text { second }\end{array}$ \\
\hline Trivial & Linear & $\begin{array}{l}\text { Optimal but not in- } \\
\text { teresting; naive }\end{array}$ & 9 second \\
\hline $\begin{array}{l}\text { Fixed end } \\
\text { reservoir }\end{array}$ & Linear & $\begin{array}{l}\text { Optimal but little } \\
\text { improvement }\end{array}$ & 9 second \\
\hline Nonlinear & Nonlinear & $\begin{array}{l}\text { Locally optimal } \\
\text { but realistic }\end{array}$ & 10 hours \\
\hline Discretized & Linear & $\begin{array}{l}\text { Optimal and realis- } \\
\text { tic }\end{array}$ & 9 second \\
\hline
\end{tabular}

regulation market. For a hydropower producer this is not a realistic case. Therefore, the purpose of this study is to suggest some modifications of the base model, which will provide more robust results. Different modeling approaches are tested and evaluated. The approach, where upward/downward market prices are discretized and available volume is bid according to that, is suggested as the most promising one.

Next step is to develop a model which considers also trading on intra-day market under the uncertainty.

\section{REFERENCES}

[1] "Renewable energy technologies:cost analysis:hydropower," tech. rep., International renewable energy agency, 2012.

[2] E. Lawrence, "Strategies and decision support systems for integrating variable energy resources in control centers for reliable grid operations," tech. rep., 2011.

[3] D. E. Castronuovo and P. L. J.A., "On the optimization of the dayly operation of a wind-hydro power plan," IEEE Transactions on Power Systems, vol. 19, 2004.

[4] C. Belanger and L. Gagnon, "Adding wind power to hydropower," Energy Policy, vol. 30, 2002.

[5] S.-E. Fleten and T. K. Kristoffersen, "Stochastic programming for optimizing bidding strategies of a Nordic hydropower producer," European Journal of Operation Research, vol. 181, 2007.

[6] S.-E. Fleten and T. K. Kristoffersen, "Short-term hydropower production planning by stochastic programming," Computers and Operations Research, vol. 35, 2008.

[7] E. Fariba and S.-E. Fleten, "Day-ahead market bidding for a nordic hydropower producer: taking the elbas market into account," Computational Management Science, vol. 8, pp. 75-101, 2011.

[8] G. Pritchard and G. Zakeri, "Market offering strategies for hydroelectric generators," Operations Research, vol. 51, 2003.

[9] G. Pritchard, A. B. Philpott, and P. Neame, "Hydroelectric reservoir optimization in a pool market," Mathematical Programming, vol. 103, 2005.

[10] D. De Ladurantaye, M. Gendreau, and J. Potvin, "Strategic bidding for price-taker hydroelectricity producer," IEEE Transactions on Power Systems, vol. 22, 2007.

[11] M. Olsson, On optimal hydropower bidding in systems with wind power. $\mathrm{PhD}$ thesis, Dept. of Electrical Engineering, KTH, 2009.

[12] J. Contreras, R. Espinola, F. J. Nogales, and A. J. Conejo, "Arima models to predict next-day electricity prices," vol. 18, no. 3, 2003.

[13] M. Olsson and L. Söder, "Modeling real-time balancing power market prices using combined sarima and markov processes," IEEE Transactions on Power Systems, vol. 23, 2008.

[14] G. E. P. Box and G. M. Jenkins, Time Series Analysis forecasting and control. HOLDEN-DAY, 1976.

[15] P. J. Brockwell and R. A. Davis, Time Series: Theory and Methods. Springer-Verlag New York Inc., 1991. 